\title{
Pulmonary hypertension, how to diagnose and who to treat?
}

\author{
J. W. Roos-Hesselink • F. Zijlstra
}

Published online: 8 November 2011

(C) The Author(s) 2011. This article is published with open access at Springerlink.com

Pulmonary arterial hypertension is a fatal disease with an estimated survival rate of only $50 \%$ after 5 years [1]. Over the last years, knowledge in the field of pulmonary hypertension has grown consistently and significantly. New diagnostic tools, as well as the efficacy of new medications and drug combinations, have been described.

How to define and diagnose pulmonary arterial hypertension is the first important issue. Should echocardiography be the method of choice? It is easy to access, cheap and noninvasive. However, the cut-off values are debatable. In the ESC guidelines of 2009 [2] an estimated peak pulmonary artery pressure of $>50 \mathrm{mmHg}$ is stated as 'pulmonary hypertension is likely', while a PAP of 37-50 is defined as 'possible PH'. However, the gold standard remains heart catheterisation. Now the definition is made using a mean pulmonary artery pressure of $25 \mathrm{mmHg}$ or more. Age and gender seem to have an important impact on the incidence and severity of PH. For instance, female patients with an atrial septal defect do present with pulmonary hypertension, while in male ASD patients pulmonary hypertension is seldom if ever seen.

In order to simplify the clinical management of patients, the classification of pulmonary hypertension was changed in 2009 [3]. Pre-capillary PH is defined as a wedge pressure $<15 \mathrm{mmHg}$ and can be divided into pulmonary arterial hypertension, $\mathrm{PH}$ due to lung disease, chronic thromboembolic $\mathrm{PH}$ or $\mathrm{PH}$ with unclear and/or multifactorial mechanisms. Post-capillary PH is defined as a wedge pressure above $15 \mathrm{mmHg}$ and is due to left-sided heart disease.

Who should be screened for pulmonary hypertension? Should all patients undergoing non-cardiac surgery by

J. W. Roos-Hesselink · F. Zijlstra ( $($ )

Erasmus MC Rotterdam,

Rotterdam, the Netherlands

e-mail: f.zijlstra.1@erasmusmc.nl tested? Echocardiography and certainly heart catheterisation are not routinely performed, but when complications arise postoperatively, pulmonary hypertension is not seldom detected and although the underlying cause may vary, the prognosis is unfavourable. One could argue that echocardiography screening preoperatively in patients over the age of 60 years may detect high-risk patients for general surgery. Patients known with pulmonary hypertension should be counselled against surgery, or if it is decided to proceed with surgery a team of surgeons, anaesthetists, intensivists and others should make a treatment plan for optimal perioperative care.

Advances in treatment of pulmonary arterial hypertension over the past 15 to 20 years have dramatically reduced the morbidity and mortality of the disease. A reduction of $43 \%$ in mortality and $61 \%$ in hospitalisations was found in a recent meta-analysis [4]. However the current medication is expensive and has major drawbacks so it is very important to treat the patients who might benefit. At the moment there are probably many patients left untreated who could benefit, for instance adult patients with $\mathrm{PH}$ associated with congenital heart disease. There is also an interesting group of patients with primary left-sided heart disease who have disproportionately high pulmonary pressures. In patients who have undergone a successful procedure for aortic or mitral stenosis the pulmonary arterial pressures may remain elevated, although the wedge pressure has normalised. The indication to treat these patients with disproportionately high pulmonary pressures remains controversial. For patients with left-sided systolic dysfunction with secondary pulmonary hypertension, no benefit of treatment was found and in fact may have a negative effect on outcome, but for the patients with disproportionately high pulmonary hypertension no studies are available as yet. 
We hope you enjoy reading this focus issue of the Netherlands Heart Journal, dedicated to several aspects of the rapidly evolving field of Pulmonary Hypertension [5-9].

Open Access This article is distributed under the terms of the Creative Commons Attribution Noncommercial License which permits any noncommercial use, distribution, and reproduction in any medium, provided the original author(s) and source are credited.

\section{References}

1. Kane GC, Maradit-Kremers H, Slusser JP, et al. Integration of clinical and hemodynamic parameters in the prediction of long-term survival in patients with pulmonary arterial hypertension. Chest. 2011;139:1263-4.
2. ESC guidelines for the diagnosis and treatment of pulmonary hypertension. European Heart Journal 2009;30:2493-2537

3. Simonneau G, Robbins I, Beghetti M, et al. Updated clinical classification of pulmonary hypertension. JACC. 2009;54:843-54.

4. Galie N, Manes A, Negro L, et al. A meta-analysis of randomized controlled trials in pulmonary arterial hypertension. Eur Heart J. 2009;30:394-403.

5. Schuuring MJ, van Riel ACMJ, Bouma BJ, et al. Recent progress in treatment of pulmonary arterial hypertension due to congenital heart disease. Neth Heart J 2011

6. Schölzel BE, Snijder RJ, Morshuis WJ, et al. Clinical worsening after pulmonary endarterectomy in chronic thromboembolic pulmonary hypertension. Neth Heart J 2011

7. Hoendermis ES. Pulmonary arterial hypertension: an update. Neth Heart J 2011

8. Pieper PG, Hoendermis ES. Pregnancy in women with pulmonary hypertension. Neth Heart J 2011

9. Schuuring MJ, Boekholdt SM, Windhausen A, et al. Advanced therapy for pulmonary arterial hypertension due to congenital heart disease: a clinical perspective in a new therapeutic era. Neth Heart J 2011 